\title{
Nanofabrication with a Helium Ion Microscope
}

\author{
Diederik Maas $^{* a}$, Emile van Veldhoven ${ }^{\mathrm{a}}$, Ping Chen ${ }^{\mathrm{b}}$, Vadim Sidorkin ${ }^{\mathrm{b}}$, \\ Huub Salemink ${ }^{\mathrm{b}}$, Emile van der Drift ${ }^{\mathrm{b}}$, and Paul Alkemade ${ }^{\mathrm{b}}$ \\ ${ }^{a}$ TNO Science and Industry, Stieltjesweg 1, Delft 2628CK, The Netherlands \\ ${ }^{\mathrm{b}}$ Kavli Institute of Nanoscience, Delft University of Technology, Lorentzweg 1, \\ Delft 2628CJ, The Netherlands
}

\begin{abstract}
The recently introduced helium ion microscope (HIM) is capable of imaging and fabrication of nanostructures thanks to its sub-nanometer sized ion probe [1,2]. The unique interaction of the helium ions with the sample material provides very localized secondary electron emission, thus providing a valuable signal for high-resolution imaging as well as a mechanism for very precise nanofabrication [3]. The low proximity effects, due to the low yield of backscattered ions and the confinement of the forward scattered ions into a narrow cone, enable patterning of ultra-dense sub- $10 \mathrm{~nm}$ structures. This paper presents various nanofabrication results obtained with direct-write, with scanning helium ion beam lithography, and with helium ion beam induced deposition.
\end{abstract}

Keywords: Helium Ion Microscopy, HIM, nanoimaging, nanofabrication, scanning helium ion beam lithography, SHIBL, lithography, helium ion beam induced deposition, HIBID

\section{INTRODUCTION}

The ever shrinking device dimensions in electronic circuitry, data storage, biological sensing and photonics are pushing nano-manufacturing into the (sub-)ten-nanometer regime[4]. Nowadays, state-of-the-art technologies, such as extremeultra-violet lithography and electron beam lithography, are facing their limits in producing fine patterns at high densities. In this paper we show that the helium ion microscope is possibly the next step forward in nanofabrication. In particular low proximity effects in combination with high resolution make scanning helium ion beam lithography (SHIBL) and helium ion beam induced processing (HIBID) outstanding candidate technologies for the next generation of nanofabrication tools. However, the He ion impact on semiconductor nano-device structures may not always be harmless [5,6] and the relatively low beam current in HIM (0.1-10 pA) limits the throughput, thus inhibiting large-scale nanofabrication. Nanofabrication technologies that have a (potentially) high throughput that may benefit from this innovative technology comprise mask patterning and repair for optical and EUV lithography, and templates fabrication for nano-imprint. Furthermore, the unique nanofabrication capabilities of the HIM offer the possibility to pioneer novel device designs that are up to several nodes ahead of the state-of-the-art as e.g. proposed in the ITRS of SEMATECH [4].

\section{HIM IMAGING FOR PROCESS CONTROL}

Figure 1 illustrates the role of metrology and detection in gaining process control in industrial applications. As the development of a novel process matures, more becomes known of the required conditions for successful production. Initially, much effort is put into analysis of failures. This in general involves off-line analysis with generic analysis tools such as mass spectrometry, optical microscopy, AFM, SEM, FIB, TEM and (as illustrated in Fig. 2) HIM. When more is known of the process, measures are taken to ensure constant output quality, either by defining and maintaining a process window or by monitoring process quality with in-line inspection tools that are tailor-made for their function and have a throughput and sampling strategy that matches with the speed of the production process.

Metrology, Inspection, and Process Control for Microlithography XXIV, edited by Christopher J. Raymond, Proc. of SPIE Vol. 7638, 763814 · (c) 2010 SPIE · CCC code: 0277-786X/10/\$18 · doi: 10.1117/12.862438 




Fig. 1 The changing role of Metrology and Detection tools in gaining process control in industrial production as a function of process maturity. Acceleration of the process development requires failure analysis tools to provide correct information on samples taken from the (pilot) production line. Helium ion microscopy is a novel surface inspection technology offering coating-free imaging with (sub-)nanometer accuracy, even on sensitive nonconductive samples.

Figure 2 displays a HIM image that was recorded to characterize the morphology of the active layer of an organic polymer solar cell (OPSC). In the OPSC project at the Holst Centre, the objective is to develop efficient bulk heterojunction polymer solar cells. The organic photovoltaic devices based on the conjugated polymer/fullerene bulk heterojunctions are highly dependent on the solid state nanoscale morphology of the two components (donor/acceptor) in the photoactive layer. The need for finely phase-separated polymer-fullerene blends is due to the limited exciton diffusion length in organic semiconductors. Typical distances over which these photo-excitations can travel within a pristine material are about 10-20 nm. In the actual devices, the scale of phase separation should be closely related to the exciton diffusion lengths in the two materials involved. The morphology can be controlled by many processing parameters during the production and does influence the final cell efficiency [7]. To optimize the production process it is important to have a high resolution imaging tool that is capable of characterizing the photo-active layer in the device. However, the sample-probe interaction may cause artifacts in the images of the sample. Until recently, the imaging tools have been limited to AFM and TEM. Helium ion microscopy (HIM) is a relative new high-resolution surface imaging technique $[1,2]$. It deploys a sub-nanometer sized spot that scans the surface of the sample with high sensitivity and high speed. The technique does not require complicated sample preparation. Sample charging is cancelled by usage of an electron flood gun, synchronized with the line scan. To study the feasibility of HIM for imaging these delicate materials a comparison study has been performed: samples containing a mixture of polymers and fullerenes have been prepared in various ways to obtain variations in morphology. The challenge for this class of samples is in obtaining high-resolution images of a non-conductive layer without the creation of artifacts due to sample preparation, charging, or due to contaminating or damaging the surface [8]. Figure 2 shows a high resolution image made by the HIM. It clearly shows the presence and distribution of fullerene agglomerates in the polymer matrix. The capability to inspect and analyze the morphology of this active layer makes HIM a powerful new and very direct imaging technique for this class of samples, thus enabling a faster development of organic polymer solar cells. 


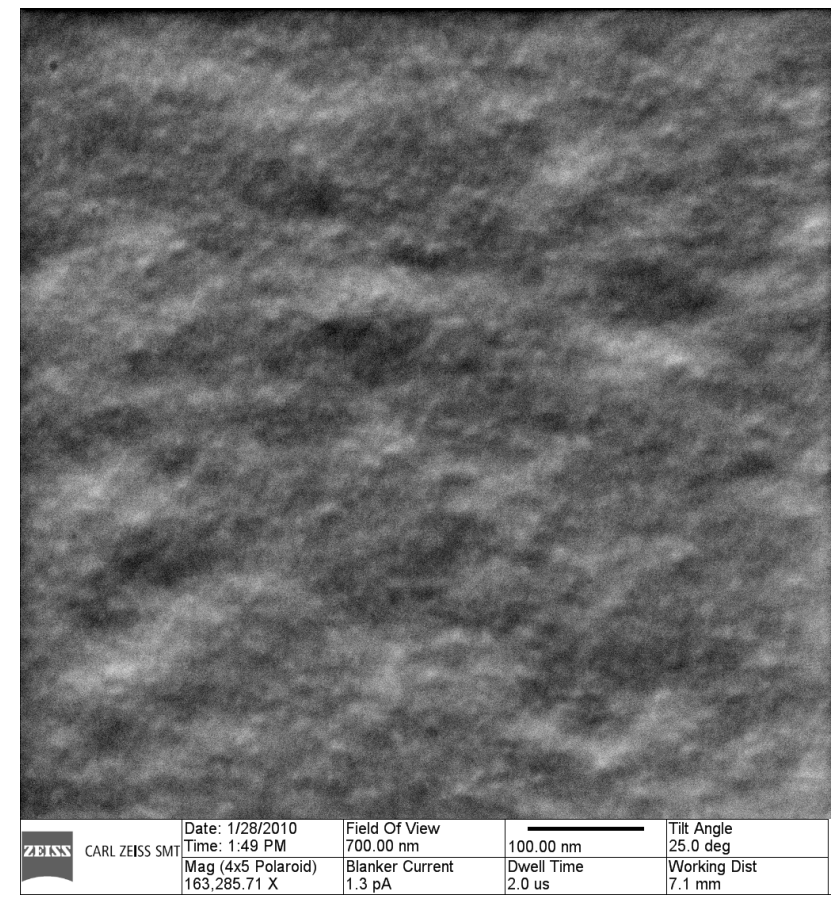

Fig. 2 High resolution HIM image of the active layer of an annealed organic polymer solar cell. The relative large features of about $100 \mathrm{~nm}$ are most likely due to the surface roughness of the sample substrate, the smaller features are an indication of how the polymers are oriented and how the fullerenes form clusters upon annealing. Sample courtesy of the Holst Centre, Eindhoven, The Netherlands.

\section{NANOFABRICATION}

This paper discusses three varieties of nanostructure fabrication with the HIM: direct write, scanning helium ion beam lithography (SHIBL), and helium ion beam induced deposition (HIBID). Each variant is discussed and illustrated in a separate paragraph below. All varieties benefit from the well-defined (sub-) nanometer sized ion probe and the extremely low proximity effect that stems from the weak back- and forward scattering of helium ions.

\subsection{Experimental}

The Carl Zeiss Orion ${ }^{\mathrm{TM}}$ Plus scanning helium ion microscope in our laboratory was equipped with an ELPHY Plus ${ }^{\mathrm{TM}}$ pattern generator from Raith and an OmniGIS ${ }^{\text {TM }}$ gas injection system (GIS) from Omniprobe. The pattern generator enables beam scan control to expose complex patterns and is deployed in the direct-write, lithography as well as the HIBID experiments. In HIBID, the focused helium ion beam interacts very locally with adsorbed precursor molecules of a specific chemistry to create a deposit with a specific shape and composition. The OmniGIS ${ }^{\mathrm{TM}}$ gas injection system device allows the control of three separate precursor gases and two carrier gases, providing wide latitude for developing HIBID process recipes. The GIS experiments discussed in this paper are limited to the Pt chemistry. The HIM was operating at 25-30 keV beam energy. The HIM and a Hitachi S4800 SEM were used for inspection of the developed structures in HSQ resist after SHIBL exposure. Variation in dose was achieved by control of the beam current and the dwell time per pixel, which varied from 20 to $2000 \mu$ s. In the HIBID experiments, dose variation was also achieved by repeating the exposures. 


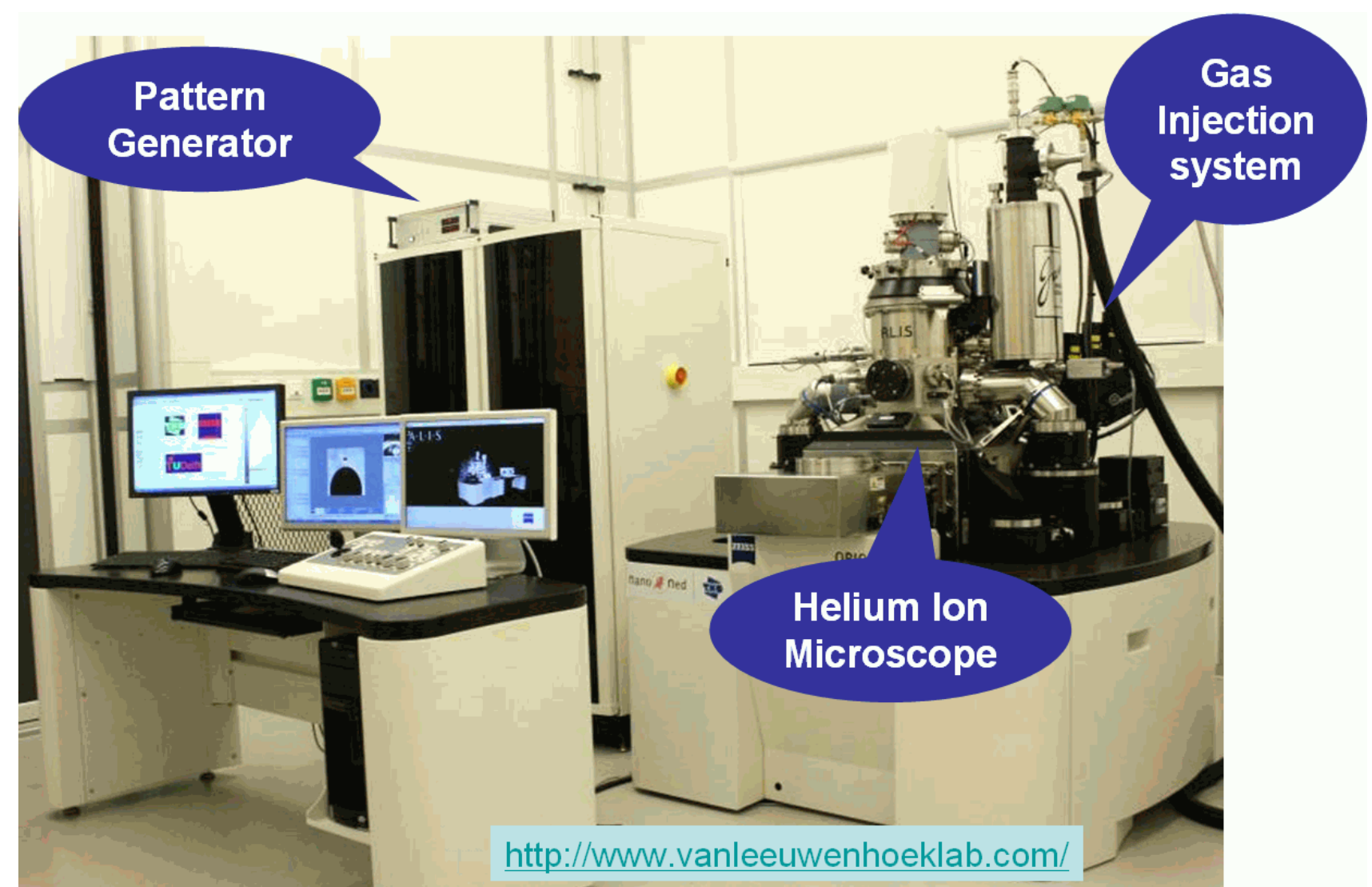

Fig. 3 The Orion ${ }^{\mathrm{TM}}$ Plus HIM equipped with a pattern generator (PG) and a gas injection system (GIS) for nanofabrication purposes in the clean room of the Van Leeuwenhoek Laboratory in Delft, the Netherlands.

\subsection{Direct write}

A recent study showed that sputtering of sub-micron sized tin balls by $30 \mathrm{keV}$ helium ions is roughly two orders of magnitude slower than by conventional gallium ions of the same energy [9]. Nevertheless, sputtering of target atoms by helium ions is one of the factors that limit the sample integrity and hence the maximal achievable signal-to-noise ratio in HIM. Figure 4 shows that helium ions can indeed cut a thin gold nano-wire: an $8 \mathrm{~nm}$ cut is made with the HIM and subsequently imaged with both HIM and TEM (inset in Fig. 4.). ${ }^{1}$ The relatively large width of the cut is partly attributed to the surface tension of the gold, which is known to cause reconfiguration after modification at the nanometer scale [10]. Earlier Au nano-wire cutting experiments using a focused $30 \mathrm{keV} \mathrm{Ga}^{+}$beam and a 200-300 $\mathrm{keV}$ STEM yielded 30 nm gaps with a not-so-well controlled shape. For the HIM cutting, the Au nano-wire synthesis process was based on a frequently used method described by Murphy et al. [11]. Cutting of gold nano-rods and nano-wires may be useful for various optoelectronic devices [12]. A key question for nano-optics is whether a nano-rod can be used as an antenna to modify the emission of a single molecule. Two groups have built such nano-antennas: Schuck et al. obtained a strong enhancement of the field with a nanometer-scale bow-tie antenna [13], while Mühlschlegel et al. reported an optical version of the simplest radio antenna: a half-wave antenna [14].

\footnotetext{
${ }^{1}$ Unpublished work of Pickard et al. (National University of Singapore) has shown that single sheet graphene can be patterned into $5 \mathrm{~nm}$ wide ribbons of significant length, using direct write with the focused beam of the HIM.
} 


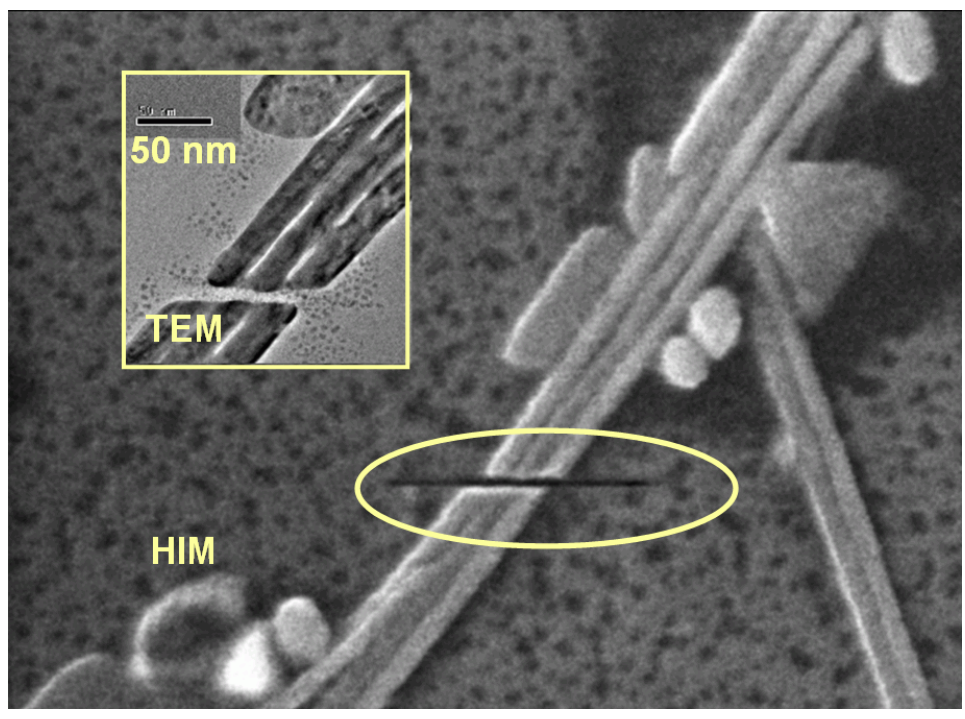

Fig. 4 HIM and TEM (inset) images of an $8 \mathrm{~nm}$ cut (in the circle) in a gold nano-wire on a carbon TEM grid. The HIM was used to navigate to the desired sample location, to cut the nano-wire using a slow line scan, and to monitor the process. Sample cleanliness was found to be important for successful cutting. The TEM image of the same area confirms the shape and size of the cut. Sample and TEM image: courtesy of H. Zandbergen, TU Delft.

\subsection{Scanning helium ion beam lithography (SHIBL)}

Over the years focused ion beam lithography (FIBL) has been a somewhat less important player in the field of nanolithography. Despite the advantages of a higher sensitivity and lower proximity effects $[15,16]$ compared to the electron beam approach, major disadvantages of the earlier FIBL work are a lower resolution due to a larger beam diameter and (certainly for $\mathrm{Ga}^{+}$ion exposure) potential damage or contamination from the ion impact or implantation [17]. Features of 12-15 nm line width in PMMA resist [18] and $30 \mathrm{~nm}$ dot size in polyphenylsilsesquioxane resist [19] have been reported with $\mathrm{Ga}^{+}$FIB. Typically, the smallest probe size of $\mathrm{Ga}^{+}$ion beams is nowadays around $5 \mathrm{~nm}$ and direct engraving in $20 \mathrm{~nm}$ thin membrane material yields nanopores down to $3 \mathrm{~nm}$ diameter [20].

Recently, we used HIM as a beam writing tool for nanolithography and compared its performance with electron beam exposure behavior [16]. The expected advantages of SHIBL over electron beam lithography (EBL) comprise reduced proximity effects and the ability to write smaller features given the sub-nanometer probe size. Altogether these characteristics greatly suppress the blurring background which arises when writing dense patterns with EBL.

Figure 5 displays images (recorded with a Hitachi S4800 SEM) of dots written with SHIBL at various pitches in a $5 \mathrm{~nm}$ thick HSQ film. Remarkably, the same average dot size of $6 \pm 1 \mathrm{~nm}$ was achieved down to a pitch of $14 \mathrm{~nm}$. This observation points to an undetectably small proximity effect for helium ion beam exposure. Several factors may be involved in this low proximity effect. Firstly, the scattering profile of helium ions is known to be confined within a narrow cone penetrating relatively deep into the material with very low backscattering [3]. Secondly, helium ions produce mostly low energy secondary electrons (SE) $(E<20 \mathrm{eV})$, which do not travel far in the resist. The results in Fig. 5 prove the $\mathrm{He}^{+}$ion beam capability for nanostructuring dense patterns with ultra-high resolution in thin HSQ resist. To our knowledge, the best results in electron beam exposure of $5 \mathrm{~nm}$ thin HSQ at present are $6 \mathrm{~nm}$ lines on a pitch of 20 $\mathrm{nm}[21]$. 




Fig. 5. SEM images of arrays of dots written in a $5 \mathrm{~nm}$ thick HSQ layer using SHIBL at pitches of a) $48 \mathrm{~nm}$, b) 24 $\mathrm{nm}$, and c) $14 \mathrm{~nm}$; and d) the average dot size vs. pitch. The insets are SEM images at a higher magnification. The average dot size for all pitches is $6 \pm 1 \mathrm{~nm}[16]$. This work illustrates the practical absence of proximity in SHIBL on such thin resists, as well as the capability for ultra-precise writing of patterns with densities up to $50 \%$.

\subsection{Helium ion beam induced deposition from the $\left(\mathrm{CH}_{3}\right)_{3} \operatorname{Pt}\left(\mathrm{C}_{\mathrm{P}} \mathrm{CH}_{3}\right)$ precursor}

Ultimate resolution and achievable pattern density in the fabrication of nanostructures have been studied also for helium ion beam induced deposition (IBID). IBID is a direct-write technology in which precursor molecules adsorbed on a substrate surface are decomposed by an ion beam induced reaction, resulting in localized material deposition [22,23]. Due to its high flexibility for the shape and location of the deposits, IBID holds much interest for prototyping threedimensional (3D) nanostructures. In this work, $\left(\mathrm{CH}_{3}\right)_{3} \mathrm{Pt}\left(\mathrm{C}_{\mathrm{P}} \mathrm{CH}_{3}\right)$ was used as precursor. The Pt deposits are inspected with HIM directly after deposition to quantify their geometry and the proximity effects.

As a first experiment, Pt pillars were grown on the native oxide of a Si wafer. Figure 6 displays HIM images recorded at 30 degrees tilt angle of series of pillars that were grown at different beam currents, but with a fixed exposure time. The deposits have a conical shape at low currents, while they consist of a cylinder with a cone on top at high currents. Obviously the pillar diameter (typically between 40 and $80 \mathrm{~nm}$ ) is not determined by the helium ion probe size. We attribute the larger pillar width to the forward scattering of the helium ions in the deposit and the scattering of the created secondary electrons that crack precursor molecules adsorbed on the pillar surface. The pillar length can easily extend into the micrometer range. Finally, it is worth mentioning that the pillars hardly have a "footing", indicating very low overspray, which suggests that the helium ion probe does not have a large tail, in contrast to e.g. the LMIS frequently used in Ga-FIB [22,24]. 




Fig. 6 HIM recording of Pt pillars, grown by helium ion beam induced deposition at various currents, but with a fixed exposure time of 3.2 seconds.

Figure 7 shows a HIM recording at 30 degrees tilt angle of a dense Pt line pattern (pitch is $50 \mathrm{~nm}$, line width $15 \mathrm{~nm}$, and line height $20 \mathrm{~nm}$ ) with a measured line edge roughness of $3 \mathrm{~nm}(3 \sigma)$. The lines seem to have a triangular shaped top, also attributed to the forward scattering of the helium ions in the deposit. The similarity in shape and dimensions of the inner and outer lines proves the absence of proximity effects in this pattern.

Figure 8 illustrates the patterning of "large" area arbitrary shapes with control over the dimensions on the nanometer scale: $e . g$. the gap between the capitals $\mathbf{T} \mathbf{N} \mathbf{O}$ is consistently between 12 and $15 \mathrm{~nm}$. This pattern was written in one go with the Raith PG with $2 \mathrm{~nm}$ pixel size, a pixel dwell time of $0.25 \mathrm{~ms}$, and a beam current of about $0.5 \mathrm{pA}$.

In conclusion, helium ion beam induced deposition in the HIM offers a promising alternative technology for the precise direct write of dense complex nanostructures. HIBID combines the main advantage of Ga-IBID (high speed) with that of electron beam deposition (high resolution). Another strong point is the ability to inspect the grown structures directly in the same instrument. Typical applications that possibly can be furthered with this technology are NEMS devices, plasmonic devices, photonic crystals, magnetic nano-domains, and templates for protein crystal growth. 


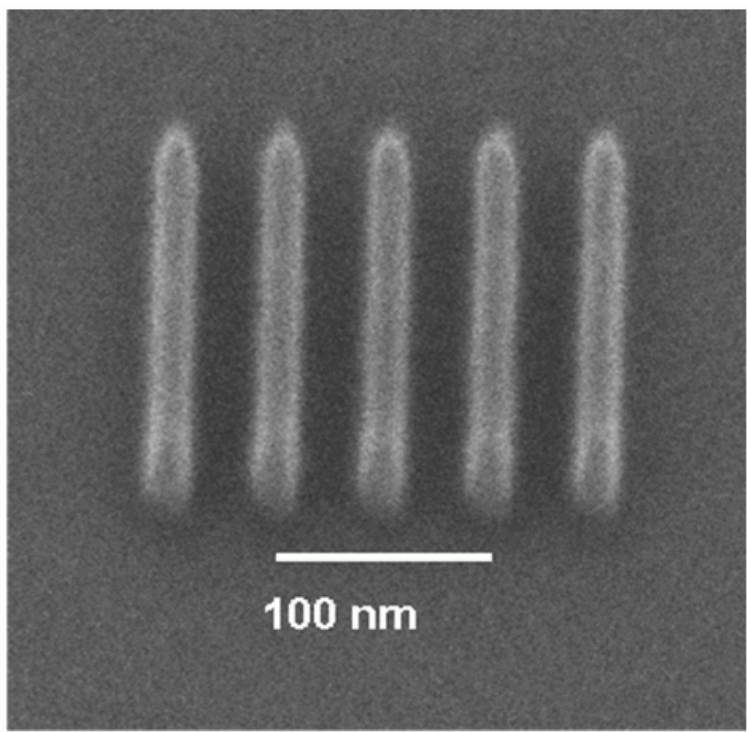

\section{Deposit shape set by} Interaction cone

Fig. 7 Platinum lines (width $15 \mathrm{~nm}$ ) at $50 \mathrm{~nm}$ pitch, deposited with HIBID and inspected with HIM at 30 degrees tilt angle. Note that the two outer lines have the same dimensions as the inner lines, demonstrating very low proximity effects. The beam energy during deposition was $25 \mathrm{keV}$, beam current was at $0.5 \mathrm{pA}$.

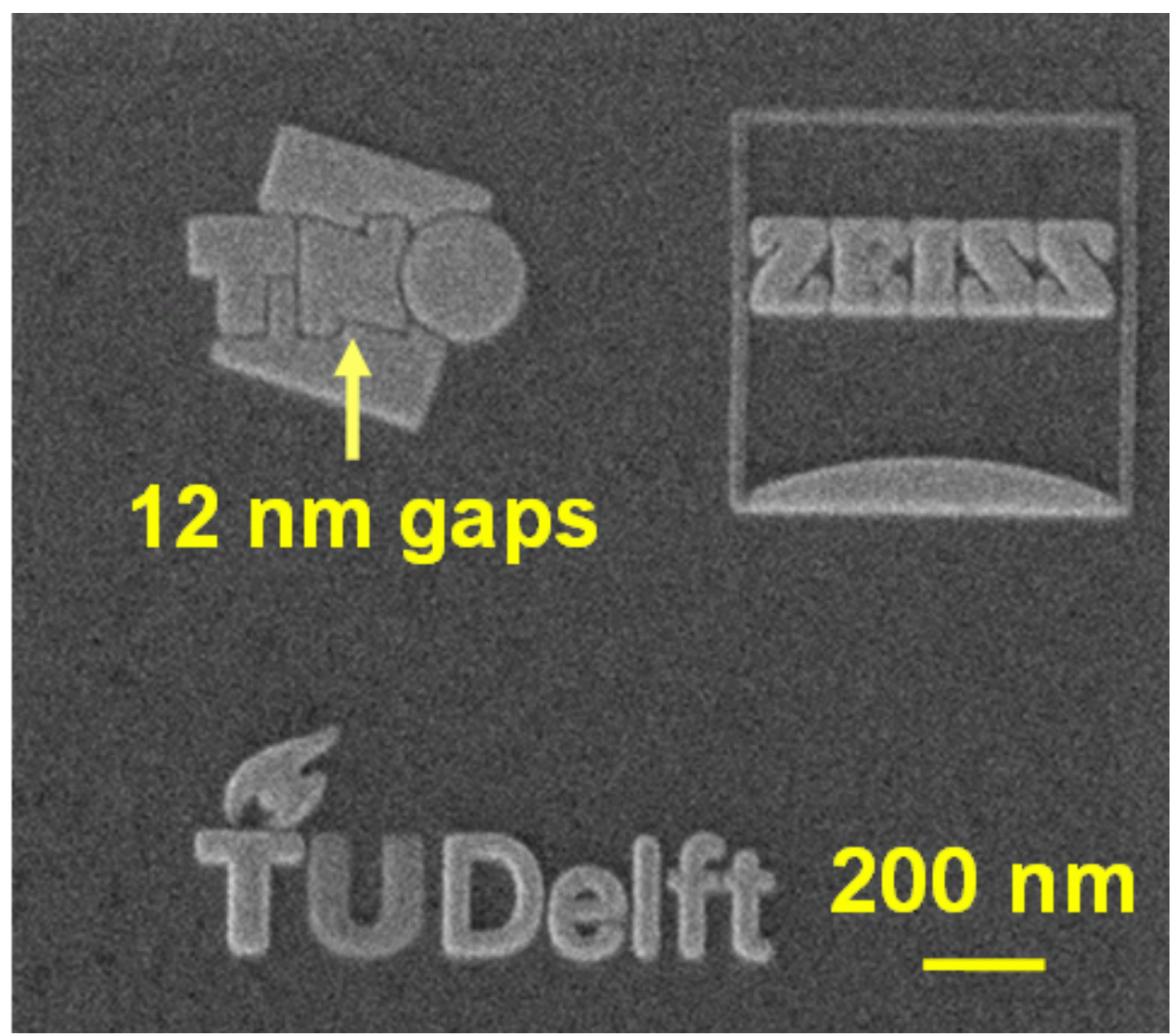

Fig. 8 Top-view HIM images of the TNO, TUD and Zeiss logo's on the native oxide layer of a silicon wafer made with Pt HIBID, demonstrating the ability to deposit "complex" dense patterns precisely. The gaps between the capitals $\mathrm{T} N \mathrm{O}$ are approximately $12-15 \mathrm{~nm}$, showing very low overspray. The beam energy during deposition was $25 \mathrm{keV}$, beam current was $0.5 \mathrm{pA}$. 


\section{CONCLUSIONS}

The helium ion microscope is a very useful new high-resolution imaging tool, especially for sensitive non-conductive samples. These samples can be imaged at extreme resolution without the need for coating of the surface. Equipping the HIM with a pattern generator and a gas injection system enables three new nanofabrication technologies: direct write, scanning helium ion beam lithography, and helium ion beam induced processing.

This paper presents nano-imaging and manufacturing application cases that exemplify the unique interaction of helium ions with the atoms in a material. Scanning $\mathrm{He}^{+}$ion beam lithography (SHIBL) on HSQ and PMMA resist layers are demonstrated to have very high resolution and superior low proximity effect as compared to electron beam lithography. In helium ion beam induced deposition (HIBID) the physical and chemical conversion of a top layer by the impinging helium ions is sufficient to grow nanostructures. HIBID from the $\left(\mathrm{CH}_{3}\right)_{3} \mathrm{Pt}\left(\mathrm{C}_{\mathrm{P}} \mathrm{CH}_{3}\right)$ precursor has shown to yield welldefined smooth pillars, narrow lines and complex dense patterns with controlled lateral dimensions down to $12 \mathrm{~nm}$. The lateral size of the HIBID deposits is determined by the scattering profile of the helium ions in the substrate and in the growing deposit, and the range of the secondary electrons created by the ions.

In summary, our initial studies with the new Orion Plus helium ion microscope at the TNO Van Leeuwenhoek Laboratory in Delft, prove that scanning helium ion beam lithography (SHIBL) and helium ion beam induced processing (HIBIP) do work. Moreover, SHIBL shows higher sensitivity and offers better resolution and has more capabilities for ultra-dense structures than electron beam lithography. In particular, nanofabrication with the HIM is expected to pay off most for the creation of ultra-high precision structures with ultra-high density and feature sizes in the sub-10 nm area, e.g. photonic crystals, templates for NIL and EUVL mask repair or patterning.

\section{ACKNOWLEDGEMENTS}

This research is part of NanoNed, a national research program on nanotechnology funded by the Dutch ministry of Economic Affairs in The Netherlands. Anja van Langen-Suurling, Hozanna Miro and Henny Zandbergen are acknowledged for their contributions to this work. Norbert Koster (TNO) is gratefully acknowledged for his clear presentation of this paper at the SPIE conference in San Jose.

*Diederik.Maas@tno.nl; phone+31 15259 2368; www.tno.nl

\section{REFERENCES}

[1] J. Morgan, J. Notte, R. Hill, and B. Ward, Microscopy Today 14, 24 (2006)

[2] A.E. Vladár, M.T. Postek and B. Ming, Microscopy Today 17, 6 (2009)

[3] R. Ramanchandra, B. Griffin and D. Joy, Ultramicroscopy 109, 748 (2009)

[4] http://www.itrs.net/

[5] E. van der Drift, R. Cheung and T. Zijlstra, Microelectron. Eng. 32, 241 (1996)

[6] R. Livengood, S. Tan, Y. Greenzweig, J. Notte and S. McVey, J. Vac. Sci. Technol. B 27, 3244 (2009)

[7] D. Veldman et al., JACS 130, 7721(2008)

[8] S. van Bavel et al. Nano Letters 9, 507 (2009)

[9] V. Castaldo, C.W. Hagen, P. Kruit, E. van Veldhoven and D.J. Maas, J. Vac. Sci. Technol. B 27, 3196 (2009)

[10] H.W. Zandbergen, R.J.H.A. van Duuren, P.F.A. Alkemade, G. Lientschnig, O. Vasquez, C. Dekker and F.D. Tichelaar, Nano Letters 5, 549 (2005)

[11] J. Murphy, N.R. Jana and L. Gearheart, J. Phys. Chem. B 105, 4065 (2001)

[12] F.M. van der Kooij, K. Kassapidou and H.N.W. Lekkerkerker, Nature 406, 868 (2000)

[13] P.J. Schuck et al., Phys. Rev. Lett. 94, 017402 (2005)

[14] P. Mühlschlegel et al., Science 308, 1607 (2005)

[15] S. Matsui, Y. Kojima, Y. Ochiai and T. Honda, J. Vac. Sci. Technol. B 9, 2622 (1991)

[16] V. Sidorkin, E. van Veldhoven, E. van der Drift, P.F.A. Alkemade, H.W.M Salemink and D.J. Maas J. Vac. Sci. Technol. B 27, L18 (2009) 
[17] D.C. Ferranti, J.C. Morgan, W.B. Thompson and W.C. Joyce, Proc. SPIE 2194, 394 (1994)

[18] R.L. Kubena, J.W. Ward, F.P. Stratton, R.J. Joyce and G.M. Atkinson, J. Vac. Sci. Technol. B 9, 3079 (1991)

[19] R.L. Kubena, R.J. Joyce, J.W. Ward, H.L. Garvin, F.P. Stratton and R.G. Brault, J. Vac. Sci. Technol. B 6, 353 (1988)

[20] J. Gierak, A. Madouri, A.L. Biance, E. Bourhis, G. Patriarche, C. Ulysse, D. Lucot, X. Lafosse, L. Auvray, L. Bruchhaus and R. Jede, Microelectron. Eng. 84, 779 (2007)

[21] A.E. Grigorescu, M.C. van der Krogt, C.W. Hagen and P. Kruit, J. Vac. Sci. Technol. B 25, 1998 (2007)

[22] I. Utke, P. Hoffmann and J. Melngailis, J. Vac. Sci. Technol. B 26, 1197 (2008)

[23] C.A. Sanford, L. Stern, L. Barriss, L. Farkas, M. Dimanna, R. Mello, D.J. Maas and P.F.A. Alkemade, J. Vac. Sci. Technol. B 27, 2660 (2009)

[24] P. Chen, H.W.M. Salemink and P.F.A. Alkemade, J. Vac. Sci. Technol. B 27, 1838 (2009) 